\title{
LATTICE ISOMORPHIC SOLVABLE LIE ALGEBRAS
}

\author{
R. C. GLAESER and B. KOLMAN 1 \\ (Received 6 February 1968; revised 9 August 1968)
}

\section{Introduction}

Let $L$ be a Lie algebra over a field $k$ of any characteristic, and consider the lattice $\mathscr{L}(L)$ of all subalgebras of $L$. In this paper we prove that if $L$ and $M$ are lattice isomorphic Lie algebras, over a field of any characteristic, and $L^{\prime}$ and $M^{\prime}$ are nilpotent, then the difference between the orders of solvability of $L$ and $M$ differs by at most one.

\section{Full intervals}

Definition. An $(n+1)$-dimensional $(n \geqq 1)$ Lie algebra is called almost abelian if it has a basis $e_{0}, e_{1}, \cdots, e_{n}$ such that $e_{0} e_{i}=e_{i}$ for $i \geqq 1$ and $e_{i} e_{j}=0$ for $i, j \geqq 1$ (cf. [3] p. 150).

Let $L$ be a Lie algebra and $A$ and $B$ subalgebras of $L$ such that $A \subseteq B$. We shall denote the lattice of all subalgebras $C$ of $L$ such that $A \subseteq C \cong B$ by $\mathscr{L}(B \div A)$.

Definition. We call a lattice $\mathscr{L}(L)$ projective if it is isomorphic to the lattice of all subspaces of a projective geometry.

Definition. An interval $\mathscr{L}(B \div A)$ of a Lie algebra $L$ is called full if every subspace $U$ of $L, A \subseteq U \subseteq B$, is a subalgebra.

Clearly, if $L$ is a Lie algebra, then $\mathscr{L}(L)$ is projective if and only if $\mathscr{L}(L \div O)$ is full.

In this paper we denote the derived algebra of a Lie algebra $L$ by $L^{\prime}$ and the derived algebra of $L^{(r-1)}$ by $L^{(r)}$. We use the symbol $\cup$ to denote the join in the lattice of subalgebras. Also, $\langle S\rangle$ is the subspace spanned by the set $S$ and $\langle U, V\rangle$ is the subspace spanned by the subsets $U$ and $V$.

Proposirion 1. For a Lie algebra $L, \mathscr{L}(L)$ is projective if and only if $L$ is abelian or almost abelian.

Proof. If $L$ is abelian or almost abelian, then clearly $\mathscr{L}(L \div O)$ is full.

1 The authors wish to thank the referee for his suggestions, which resulted in shortening several of the proofs. 
Conversely, suppose that $\mathscr{L}(L \div O)$ is full and that $L$ is not abelian. Then there exists a two dimensional non-abelian subalgebra of $L$. Hence, there exist $e, x \in L$ such that $e x=x \neq o$. Now suppose that $e, x$ and $y$ are linearly independent. Then $e y=\lambda e+\mu y$ for some $\lambda, \mu$ in the field, and

$$
e(x+y)=x+\lambda e+\mu y \in\langle e, x+y\rangle .
$$

It then follows that $\mu=1$ and that $e(e y)=e y$. Thus, $L=\langle e, e L\rangle$. Since $e L$ is a subalgebra we conclude that $L^{\prime}=e L$.

Now $(e+x) x=x \neq 0$, and so by the above $(e+x) L=L^{\prime}$ and $(e+x) y=y$ for all $y \in L^{\prime}$. But $e y=y$ for $y \in L^{\prime}$, and thus $x y=0$. It then follows that $L$ is almost abelian. This completes the proof.

It is well known that in a nilpotent Lie algebra $L, L^{\prime}=\Phi(L)$, the Frattini subalgebra. If $\mathscr{L}(L \div A)$ is full, then $A$ is an intersection of maximal subalgebras, and hence $A \supseteqq \Phi(L)=L^{\prime}$. Therefore, a nilpotent Lie algebra $L$ is abelian if and only if $\mathscr{L}(L)$ is projective. Also, if $L$ is a nilpotent Lie algebra with subalgebras $A$ and $B, A \cong B$, and if $\mathscr{L}(B \div A)$ is full then $B^{\prime} \subseteq A$.

Lemma 1. Let $L$ and $M$ be solvable Lie algebras and let $\varphi: \mathscr{L}(L) \rightarrow \mathscr{L}(M)$ be a lattice isomorphism. If $A$ and $B$ are subalgebras of $L$ such that $A \cong B$ and $\mathscr{L}(B \div A)$ is full then $\mathscr{L}(\varphi(B) \div \varphi(A))$ is full.

Proof. Let $V$ be a subspace of $M$ such that $\phi(A) \leqq V \leqq \varphi(B)$. Let $x, y \in V$, we show that $x y \in V$. Since $\langle x\rangle,\langle y\rangle$ are subalgebras of $M$, there exist $x_{0}, y_{0} \in L$ such that $\varphi\left(\left\langle\boldsymbol{x}_{\mathbf{0}}\right\rangle\right)=\langle x\rangle$ and $\varphi\left(\left\langle y_{\mathbf{0}}\right\rangle\right)=\langle y\rangle$. Let $U=\left\langle x_{0}, y_{0}, A\right\rangle$. Then $A \subseteq U \subseteq B$ and so by assumption $U$ is a subalgebra of $L$. Thus, $U=\left\langle x_{0}\right\rangle \cup\left\langle y_{0}\right\rangle \cup A$. Since $L$ and $M$ are solvable, $\varphi$ preserves dimensions. From $\operatorname{dim} A=\operatorname{dim} \varphi(A)$ it follows that

$$
\operatorname{dim}\left\langle x_{0}, y_{0}, A\right\rangle=\operatorname{dim}\langle x, y, \varphi(A)\rangle .
$$

But $\operatorname{dim} U=\operatorname{dim} \varphi(U)$ and therefore $\varphi(U)=\langle x, y, \varphi(A)\rangle \subseteq V$. Thus, $x y \in V$.

\section{Order of solvability}

THEOREM 1. If $L$ and $M$ are lattice isomorphic nilpotent Lie algebras, then $L$ and $M$ have the same order of solvability.

Proof. Since $L / L^{\prime}$ is abelian, we have that $\mathscr{L}\left(L / L^{\prime}\right)$ is projective, which implies that $\mathscr{L}\left(L \div L^{\prime}\right)$ is full. If $\varphi$ is the lattice isomorphism between $\mathscr{L}(L)$ and $\mathscr{L}(M)$ we then have that $\mathscr{L}\left(M \div \varphi\left(L^{\prime}\right)\right)$ is full and hence $\varphi\left(L^{\prime}\right) \supseteqq M^{\prime}$. Similarly, $\varphi^{-1}\left(M^{\prime}\right) \supseteqq L^{\prime}$. Thus, $M^{\prime}=\varphi\left(L^{\prime}\right)$. By induction, $M^{(k)}=\varphi\left(L^{(k)}\right)$, which implies that $L$ and $M$ have the same order of solvability. 
Remark. We also note that Theorem 1 follows from Corollaries $1^{\prime}$ and $2^{\prime}$ on pages 458 and 459 of [2].

THEOREM 2. Let $L$ and $M$ be lattice isomorphic Lie algebras, with $L^{\prime}$ and $M^{\prime}$ nilpotent. Then the orders of solvability of $L$ and $M$ differ by at most one.

Proor. Let $\varphi$ be the lattice isomorphism between $\mathscr{L}(L)$ and $\mathscr{L}(M)$. Now $\varphi\left(L^{\prime}\right) / \varphi\left(L^{\prime}\right) \cap M^{\prime}$ is abelian for it is isomorphic to $\varphi\left(L^{\prime}\right) \cup M^{\prime} \mid M^{\prime}$. Therefore, $\mathscr{L}\left(\varphi\left(L^{\prime}\right) \div \varphi\left(L^{\prime}\right) \cap M^{\prime}\right)$ is full. By Lemma 1,

$$
\mathscr{L}\left(L^{\prime} \div L^{\prime} \cap \varphi^{-1}\left(M^{\prime}\right)\right)
$$

is full. Since $L^{\prime}$ is nilpotent,

Similarly,

$$
L^{\prime \prime} \cong L^{\prime} \cap \varphi^{-\mathbf{1}}\left(M^{\prime}\right) \subseteq L^{\prime} .
$$

$$
M^{\prime \prime} \cong M^{\prime} \cap \varphi\left(L^{\prime}\right) \subseteq M^{\prime}
$$

Now $L^{\prime} \cap \varphi^{-1}\left(M^{\prime}\right)$ and $\varphi\left(L^{\prime}\right) \cap M^{\prime}$ are lattice isomorphic. By Theorem 1 they have the same order of solvability, say $r$. We then have

and

$$
L^{(r)}=\left(L^{\prime}\right)^{(r-1)} \supseteqq\left(L^{\prime} \cap \varphi^{-1}\left(M^{\prime}\right)\right)^{(r-1)} \neq O,
$$

$$
L^{(r+2)} \cong\left(L^{\prime} \cap \varphi^{-1}\left(M^{\prime}\right)\right)^{(r)}=0 .
$$

Thus, the order of solvability of $L$ is either $r+1$ or $r+2$. Similarly, we find that $M^{(r)} \neq O$ and $M^{(r+2)}=O$, which implies that the order of solvability of $M$ is either $r+1$ or $r+2$. This completes the proof.

CoRollary 1. If $L$ and $M$ are lattice isomorphic solvable Lie algebras over a field of characteristic zero, then the orders of solvability of $L$ and $M$ differ by at most one.

\section{References}

[1] Barnes, D. W., 'Lattice Isomorphisms of Lie Algebras', J. A ustral. Math. Soc. 4 (1964), $470-475$.

[2] Barnes, D. W., and Wall, G. E., 'On Normalizer Preserving Lattice Isomorphisms Between Nilpotent Groups', J. Austral. Math. Soc. 4 (1964), 454-69.

[3] Kolman, B., 'Semi-modular Lie Algebras', J. Sci. Hiroshima Univ. Ser. A-I 29 (1965), $149-63$.

Temple University, Philadelphia, Pa.

Drexel Institute of Technology, Philadelphia, $\mathrm{Pa}$. 